\title{
A Programme for Risk Assessment and Minimisation of Progressive Multifocal Leukoencephalopathy Developed for Vedolizumab Clinical Trials
}

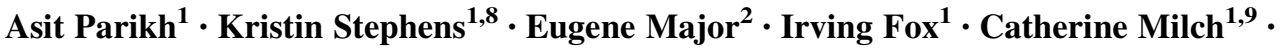 \\ Serap Sankoh ${ }^{1,10} \cdot$ Michael H. Lev ${ }^{3} \cdot$ James M. Provenzale $^{4} \cdot$ Jesse Shick $^{5,11}$. \\ Mark Patti $^{1} \cdot$ Megan McAuliffe $^{1,12} \cdot$ Joseph R. Berger $^{6,13} \cdot$ David B. Clifford $^{7}$
}

Published online: 8 May 2018

(C) The Author(s) 2018

\begin{abstract}
Introduction Over the past decade, the potential for drugassociated progressive multifocal leukoencephalopathy (PML) has become an increasingly important consideration in certain drug development programmes, particularly those of immunomodulatory biologics. Whether the risk of PML with an investigational agent is proven (e.g. extrapolated from relevant experience, such as a class effect) or merely theoretical, the serious consequences of acquiring PML require careful risk minimisation and assessment. No single standard for such risk minimisation exists. Vedolizumab is a recently developed monoclonal antibody to $\alpha 4 \beta 7$ integrin. Its clinical development necessitated a dedicated PML risk minimisation assessment as part of a global preapproval regulatory requirement.
\end{abstract}

Electronic supplementary material The online version of this article (https://doi.org/10.1007/s40264-018-0669-8) contains supplementary material, which is available to authorized users.

Asit Parikh

asit.parikh@takeda.com

1 Takeda Pharmaceuticals International Co., 40 Landsdowne Street, Cambridge, MA 02139, USA

2 National Institute of Neurological Disorders and Stroke, National Institutes of Health, Bethesda, MD, USA

3 Massachusetts General Hospital, Boston, MA, USA

4 Duke University Medical Center, Durham, NC, USA

5 Takeda Pharmaceuticals International, Inc, Deerfield, IL, USA

6 University of Kentucky, Lexington, KY, USA

7 Washington University School of Medicine, St. Louis, MO, USA
Objective The aim of this study was to describe the multiple risk minimisation elements that were incorporated in vedolizumab clinical trials in inflammatory bowel disease patients as part of the risk assessment and minimisation of PML programme for vedolizumab.

Methods A case evaluation algorithm was developed for sequential screening and diagnostic evaluation of subjects who met criteria that indicated a clinical suspicion of PML. An Independent Adjudication Committee provided an independent, unbiased opinion regarding the likelihood of PML.

Results Although no cases were detected, all suspected PML events were thoroughly reviewed and successfully adjudicated, making it unlikely that cases were missed. Conclusion We suggest that this programme could serve as a model for pragmatic screening for PML during the clinical development of new drugs.

8 Present Address: Syros Pharmaceuticals, Cambridge, MA, USA

9 Present Address: Eli Lilly and Company, Indianapolis, IN, USA

10 Present Address: Syndax Pharmaceuticals, Waltham, MA, USA

11 Present Address: Gilead Sciences, Foster City, CA, USA

12 Present Address: Biogen, Cambridge, MA, USA

13 Present Address: University of Pennsylvania, Philadelphia, PA, USA 


\section{Key Points}

PML risk minimisation is a consideration in many drug development programmes involving investigational or approved immunomodulatory agents.

This programme for Risk Assessment and Minimisation of PML (RAMP) demonstrated that practical and effective screening and surveillance measures can be applied successfully in clinical trials to help minimise and assess the potential risk of PML associated with new drugs.

No cases of PML were identified during the development of vedolizumab following rigorous risk minimisation and assessment via the RAMP.

The overall number of new unexplained neurological events detected, and the number of costly or invasive procedures (i.e. magnetic resonance imaging, lumbar puncture) required by the stepwise evaluation were low in an inflammatory bowel disease population.

\section{Introduction}

Progressive multifocal leukoencephalopathy (PML) is a rare but serious demyelinating opportunistic infection of the central nervous system caused by the JC polyomavirus. This virus is near ubiquitous, with approximately 55-60\% of healthy adults harbouring a clinically latent infection. However, PML rarely develops in immunocompetent hosts [1-3]. The infection was originally recognised for its association with severe immunocompromise, such as occurs with haematologic malignancies, human immunodeficiency virus infection/acquired immune deficiency syndrome and solid organ transplantation [4]. More recently, PML has also been reported in association with a number of immunosuppressive agents used to treat immune-mediated diseases and cancer, [2, 5-10], particularly monoclonal antibodies directed against cell surface integrins [11-13]. In 2005, three cases of PML were reported in clinical trials of natalizumab, among approximately 3000 patients, with a mean latency of 18 months [14-16]. The findings were surprising in light of the safety profile of natalizumab up to that point, and the absence of a prior association between its mechanism of action (i.e. antibody against $\alpha 4$ integrin) and PML. Efalizumab, a monoclonal antibody against $\alpha \mathrm{L} \beta 2$ integrin, was also shown to have an association with PML, which ultimately led to its withdrawal from the market [17]. Risk estimates of integrin therapeutics appeared to be elevated, even when compared with other classes of drugs associated with PML. These facts raised the possibility that other integrin antagonists that target the immune system might also be associated with an increased risk, necessitating appropriate PML risk minimisation and assessment measures in clinical trials [18]. However, the rarity of PML, along with its varied clinical presentation, render early diagnosis challenging. Currently, no validated tools exist to systematically screen for PML; therefore, systematic approaches toward PML risk minimisation with early detection of possible cases, both in clinical trials and other clinical settings, are needed. In this study, we describe the Risk Assessment and Minimisation of PML (RAMP) programme, designed to address this need. The RAMP was created for, and implemented during, the clinical development of vedolizumab, a monoclonal antibody to $\alpha 4 \beta 7$ integrin for the treatment of inflammatory bowel disease (IBD), ulcerative colitis (UC) and Crohn's disease (CD).

In creating the RAMP to support vedolizumab development, it was unclear if an approach toward proactive risk minimisation could be validated in the absence of actual PML events. The exclusive binding of vedolizumab to integrin $\alpha 4 \beta 7$ inhibits the adhesion of certain types of $B$ and $\mathrm{T}$ lymphocytes to mucosal addressin cell adhesion molecule (MAdCAM-1), which is primarily expressed on gut vascular endothelial cells. This fact made it unlikely that vedolizumab would result in general immunosuppression or an increased PML risk [19, 20].

Although cases of PML have been initially detected radiographically, diagnosis is most often based on clinical suspicion in at-risk patients who exhibit symptoms (typically a change in neurological symptoms or function). Thereafter, a confirmatory evaluation typically includes a combination of the following: brain magnetic resonance imaging (MRI) revealing characteristic white matter lesions, and either lumbar puncture with detection of JC viral DNA in cerebrospinal fluid (CSF) by polymerase chain reaction (PCR) or biopsy evidence of JC virus in brain tissue [21]. For these reasons, the RAMP focused on patient and study site staff education about the presenting signs and symptoms of PML through standardised teaching materials. The programme also utilised screening questionnaires to monitor PML risk throughout a patient's time on-study, and a stepwise management algorithm for potential cases. When cases of persistent, new, unexplained neurological symptoms arose, they were adjudicated by a panel of experts with extensive expertise in diagnosing PML (all co-authors of this report).

In this study, we present the cumulative experience involving approximately 3000 patients with $\mathrm{UC}$ or CD over the course of 7 years of vedolizumab clinical development. From these data, we propose that the RAMP constitutes a 
staged, pragmatic risk management tool for use in clinical trials of investigational agents for which the possibility of drug-associated PML represents an important consideration.

\section{Methods}

\subsection{Studies and Patients}

The RAMP was implemented in six vedolizumab phase II and III clinical trials in patients with UC or CD (Table 1). These studies were conducted at approximately 300 study sites in nearly 40 countries in North America, Europe, Asia, Africa, and Australia.

Prior to study enrolment, informed consent regarding a potential risk of PML associated with administration of the study drug was obtained. The text of the informed consent form can be found in Electronic Supplementary Material 2.

Protocol eligibility criteria, including PML checklists (described below), were utilised to screen for patients who were either deemed to be at increased risk of PML based on a set of predefined criteria, or had other neurological diagnoses that would render an evaluation of PML more difficult, e.g. multiple sclerosis.

\subsection{Subject and Staff Education}

Patients were provided with a brochure written in lay language describing the risks of PML. as well as the presenting neurological symptoms for which to remain vigilant (Electronic Supplementary Material 3). Patients were also given a wallet card, which concisely summarised the information in the brochure, for portability and easy reference (Electronic Supplementary Material 4).

Study staff (including investigators, nurses, and clinical study coordinators) were trained on the pathophysiology and natural history of PML, including signs and symptoms of disease. They were also educated on the importance of the cessation of administration of vedolizumab and the expedited evaluation of suspected PML cases when suspicion of PML was high. A brochure suitable for medical personnel containing detailed technical language was provided to each member of the study staff (Electronic Supplementary Material 5). The principal investigators were generally gastroenterologists, rather than neurologists. For that reason, investigator training included a video demonstration of how to perform a targeted neurological examination to evaluate common clinical presentations of PML (e.g. aphasia, limb weakness, etc.). The video was provided to all investigators in DVD format for quick reference.

We required a local neurologist to be included as a subinvestigator at each clinical study site; however, PML is an uncommon disease and even experienced neurologists lack first-hand familiarity with the acute clinical presentation. For this reason, we trained the local neurologist on the important clinical features that might indicate a PML diagnosis, and provided the study protocol in advance of initiating the trial.

Table 1 RAMP programme implementation: multiple-dose studies in patients with IBD $^{\text {a }}$

\begin{tabular}{|c|c|c|c|}
\hline Study & Phase & Design & Patients \\
\hline $\begin{array}{c}\mathrm{C} 13002 \\
{[29]}\end{array}$ & II & $\begin{array}{l}\text { Double-blind, randomised, } \\
\text { placebo-controlled }\end{array}$ & $\begin{array}{l}\text { Had active UC and were receiving stable doses of oral 5-aminosalicylates, corticosteroids, } \\
\text { and/or purine antimetabolites or methotrexate }(n=46)\end{array}$ \\
\hline $\begin{array}{c}\mathrm{C} 13004 \\
{[30]}\end{array}$ & II & Open-label, long-term & $\begin{array}{l}\text { Had active UC and were treatment-naive, had participated in study } \mathrm{C} 13002 \text {, or had CD and } \\
\text { were treatment-naive }\left(N=72 ;^{\mathrm{b}} 53 \mathrm{UC} \text { patients and } 19 \mathrm{CD} \text { patients) }\right.\end{array}$ \\
\hline $\begin{array}{c}\mathrm{C} 13006 \\
{[31]}\end{array}$ & III & $\begin{array}{l}\text { Double-blind, randomised, } \\
\text { placebo-controlled }\end{array}$ & $\begin{array}{l}\text { Had moderately to severely active UC with documented failure or intolerance to one or } \\
\text { more corticosteroids, immunosuppressives (i.e. azathioprine/6-mercaptopurine), or TNF } \\
\text { antagonists }(n=895)\end{array}$ \\
\hline $\begin{array}{c}\mathrm{C} 13007 \\
{[32]}\end{array}$ & III & $\begin{array}{l}\text { Double-blind, randomised, } \\
\text { placebo-controlled }\end{array}$ & $\begin{array}{l}\text { Had moderately to severely active } \mathrm{CD} \text { and were unresponsive or intolerant to one or more } \\
\text { of the following: corticosteroids, immunosuppressives (i.e. azathioprine/6- } \\
\text { mercaptopurine/methotrexate), or TNF antagonists }(n=1115)\end{array}$ \\
\hline $\begin{array}{c}\mathrm{C} 13011 \\
{[33]}\end{array}$ & III & $\begin{array}{l}\text { Double-blind, randomised, } \\
\text { placebo-controlled }\end{array}$ & $\begin{array}{l}\text { Had moderately to severely active } \mathrm{CD} \text {, and most had previous TNF-antagonist failure } \\
\quad(n=416)\end{array}$ \\
\hline $\begin{array}{l}\mathrm{C} 13008 \\
{[34,35]}\end{array}$ & III & Open-label, long-term & $\begin{array}{l}\text { Had moderately to severely active UC or CD, and either participated in studies C13004, } \\
\text { C13006, C13007 or C13011 or were treatment-naive }(n=2244)\end{array}$ \\
\hline
\end{tabular}

$\overline{C D}$ Crohn's disease, $T N F$ tumour necrosis factor, $U C$ ulcerative colitis, $P M L$ progressive multifocal leukoencephalopathy, $I B D$ inflammatory bowel disease, RAMP Risk Assessment and Minimisation for PML

${ }^{a}$ PML checklist findings were not summarised for the five studies in healthy volunteers because of limited drug exposure (i.e. one dose) and a lack of concomitant risk factors

${ }^{\mathrm{b}}$ Patients from the C13002 study could rollover into this study. Of the 53 UC patients, 38 rolled over and 19 were treatment-naive; all 19 CD patients were treatment-naive 
At investigator meetings and mid-study refresher webbased conferences, study staff were also trained on the PML checklists (described below) and the PML case evaluation algorithm, which were to be used at each patient visit to systematically evaluate and triage new, unexplained neurological findings.

\subsection{Progressive Multifocal Leukoencephalopathy (PML) Checklists, PML Case Evaluation Algorithm, and Serum JC Virus Assay}

An Independent Adjudication Committee (IAC) was formed with the purpose of providing an independent, unbiased opinion regarding the likelihood of PML in a subject who developed new neurological symptoms or signs, or who otherwise met criteria that indicated a clinical suspicion of PML. The subjective PML checklist and objective PML checklist can be found in Electronic Supplementary Material 6 , and a description of how IAC experts were identified and the charter describing roles and responsibilities, process flow, and scoring systems for case evaluation can be found in Electronic Supplementary Material 2.

The IAC, together with the sponsor, created a PML case evaluation algorithm for sequential screening and diagnostic evaluation (Fig. 1). This algorithm follows a stepwise series of measures to diagnose PML, including brain MRI and lumbar puncture. A detailed description of the algorithm and its individual steps can be found in Electronic Supplementary Material 2.

Following publication of an American Association of Neurology (AAN) consensus position on diagnostic criteria for PML in 2013, these criteria were adopted into the RAMP to define PML cases [21].

Blood-based assessment of JC viral DNA by PCR was initially performed as an exploratory biomarker for PML risk based on the most current state of understanding in 2007. Sera were collected longitudinally and stored, then assessed retrospectively in batches. However, over the course of vedolizumab clinical development, this potential biomarker was found to lack predictive value [22]; hence, following discussion with key regulatory authorities, that assessment was discontinued. The JCV serologic assay, developed subsequently and ultimately proven to have predictive value, was not available during our investigation [23].

\subsection{Adjudication of Potential Cases}

IAC neuroradiologists were asked to utilise a 0-5 Likert scale ( 5 being highest) to assign the probability of PML based on the imaging findings (see Electronic Supplementary Material 1: Table S1). In the event of a discrepancy, the neuroradiologists were to confer and assign a single score.
Within $48 \mathrm{~h}$ of receiving the data, IAC members were expected to record their reviews on evaluation worksheets that they forwarded to the IAC chairperson, who then collated the judgements in these reviews and provided a final assessment of the case to the sponsor, documenting key findings and assessing the likelihood of PML based on the qualitative scale below early in the programme, and the AAN consensus criteria later in the programme.

- Not PML.

- Possible PML. More information is needed to exclude or confirm diagnosis.

- Probable PML. Very strong clinical suspicion in the absence of CSF PCR results and brain histopathology results.

- Definite PML. Positive CSF PCR analysis for JC virus or confirmed brain histopathology.

If the assessment could not be completed based on incomplete data, additional data were requested from the study site. Once all IAC members had performed their analysis, the IAC Chairperson provided a signed memorandum to the sponsor with a brief synopsis of key findings and the consensus assessment, either making a determination of PML or not PML, or requesting additional information. The IAC recommendations were not considered binding given the fact that clinical care was intended to occur according to local standards.

\subsection{Post-Study Telephone Follow-Up}

Once patients concluded study participation, they were contacted at 6-month intervals, for a period of 2 years, by a telephone call centre, a Clinical Research Organisation $(\mathrm{CRO})$ representative or the enrolling site, to be asked focused questions about their health, including occurrence of surgery, hospitalisation, serious infection, cancer and clinical features suggestive of PML (see Electronic Supplementary Material 7). A minimum of four attempts at telephone calls were made to reach each patient at each time point, even if attempts at prior time points had been unsuccessful. The sponsor received email alerts for any positive responses.

\section{Results}

\subsection{Checklist Findings}

The vedolizumab phase III clinical development programme spanned 7 years and included 2884 patients (1114 with UC; 1770 with CD). The mean duration of exposure was approximately 450 days per patient (range 0-1865). Exposure duration of $>2$ years and prior and current immunosuppressive use are well-recognised risk factors for 


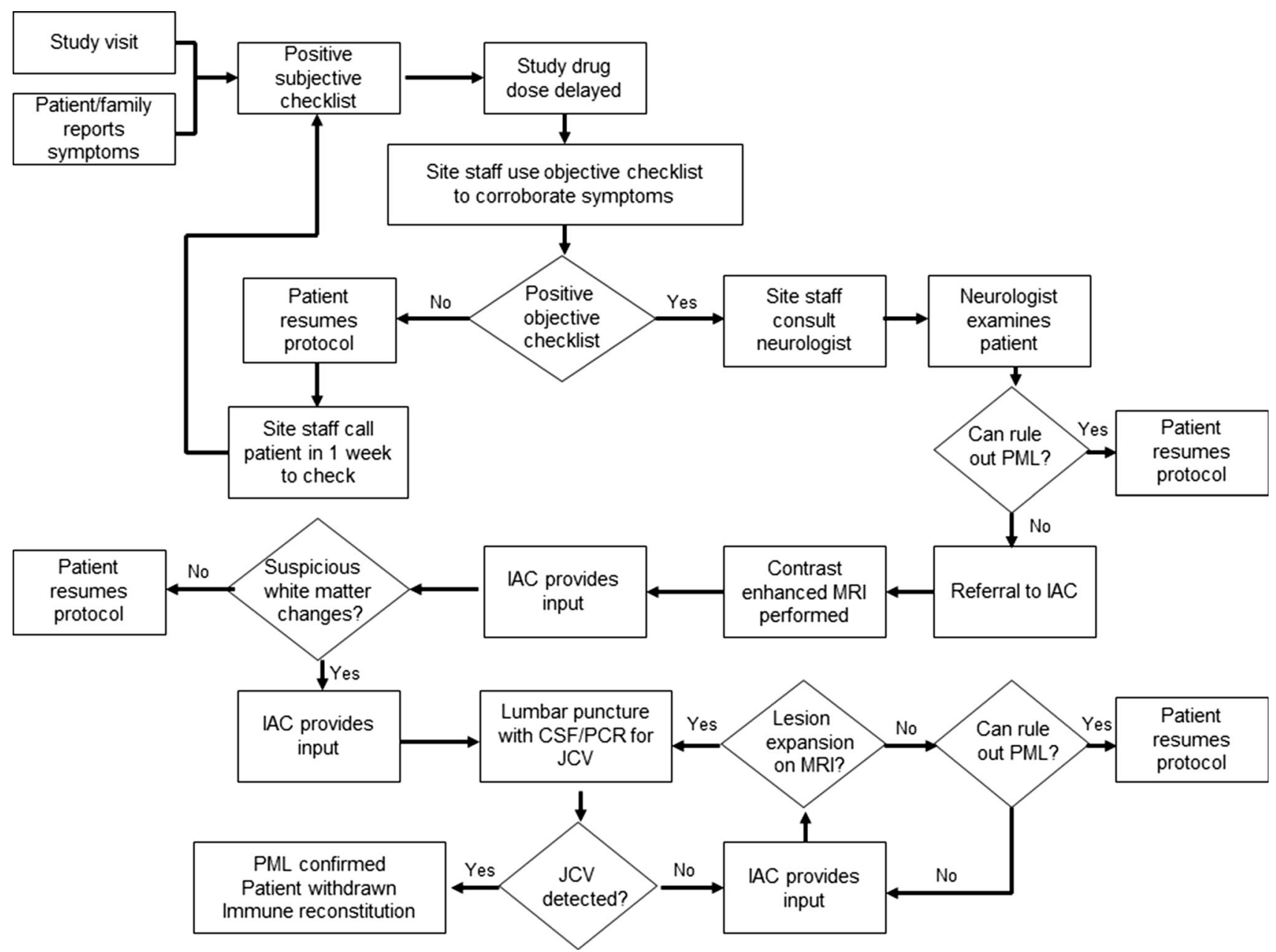

Fig. 1 RAMP programme case evaluation algorithm. CSF cerebrospinal fluid, IAC Independent Adjudication Committee, JCV JC virus, MRI magnetic resonance imaging, $P C R$ polymerase chain reaction

PML with natalizumab. A total of 835 vedolizumab patients had over 2 years of exposure, approximately $80 \%$ had prior immunosuppressive use (thiopurines, methotrexate), and $30 \%$ had current use.

As of March 2013, 2913 patients (UC: 1142; CD: 1771) (Table 2) completed 57,986 subjective checklists (UC:
24,919; CD: 33,067). Positive subjective findings led to completion of 167 and 342 objective checklists in 95 UC patients and $189 \mathrm{CD}$ patients, respectively. Very few patients $(<1.0 \%)$ indicated a positive response at baseline for the subjective checklist questions; those who did were excluded from enrolling. All neurologic signs and
Table 2 Summary of PML checklist results

\begin{tabular}{llll}
\hline & UC & CD & Total \\
\hline Patients with a subjective PML checklist administered $[n(\%)]^{\mathrm{a}}$ & $n=1142$ & $n=1771$ & $n=2913$ \\
Positive subjective findings & $95(8)$ & $189(11)$ & $284(10)$ \\
Objective PML checklist administered & $95(8)$ & $189(11)$ & $284(10)$ \\
Abnormal objective findings & $17(1)$ & $45(3)$ & $62(2)$ \\
Subjective checklists administered $[n(\%)]$ & $n=24,919$ & $n=33,067$ & $n=57,986$ \\
Positive subjective findings & $167(<1)$ & $342(1)$ & $509(<1)$ \\
Objective PML checklist administered & $165(<1)$ & $341(1)$ & $506(<1)$ \\
Abnormal objective findings & $18(<1)$ & $51(<1)$ & $69(<1)$ \\
\hline
\end{tabular}

$C D$ Crohn's disease, $U C$ ulcerative colitis, $P M L$ progressive multifocal leukoencephalopathy

${ }^{a}$ Proportions are based on the number of patients who completed one or more subjective checklists 
Fig. 2 Positive subjective checklist findings: signs and symptoms ${ }^{1}$. ${ }^{1}$ Subjective checklist symptoms: gait/coordination (e.g. bumping into objects, difficulty writing); confusion (e.g. problems with memory/thinking); vision/ ocular motility (e.g. difficulty reading); sensation (e.g. loss, numbness); speaking (e.g. dysarthria, aphasia); comprehension (e.g. inability to follow serial commands); weakness/spasticity (e.g. pronator drift, lack of muscle strength)

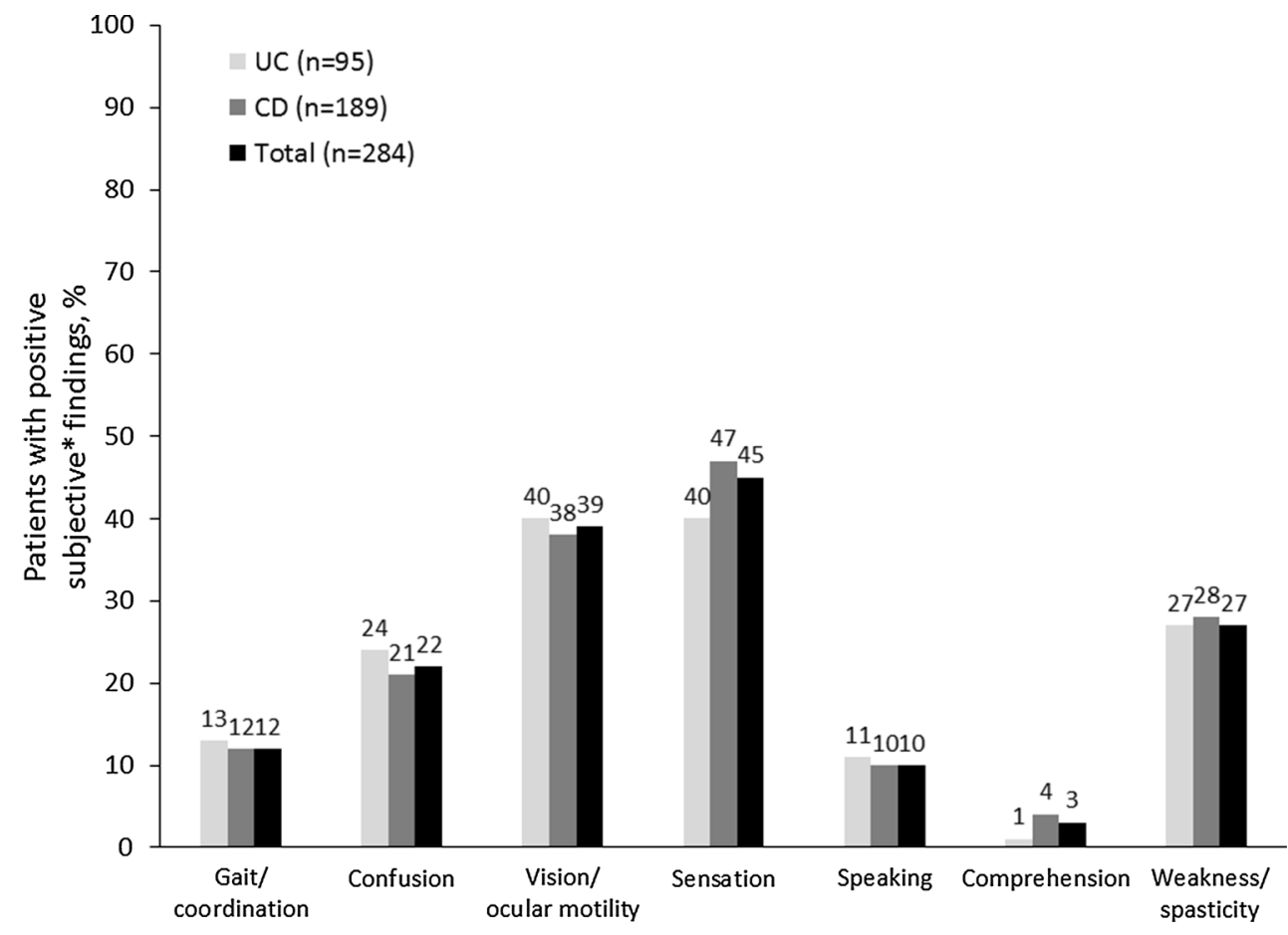

symptoms that led to positive subjective findings or abnormal objective findings are shown in Fig. 2. Positive responses were most commonly sensory in origin (e.g. paraesthesias, numbness, etc.), followed by visual findings and limb weakness less commonly reported.

\subsection{Algorithm Utilisation}

The IAC evaluated suspected cases involving 83 patients (UC: 24; CD: 59) (Table 3). Adjudication was achieved within 7 days for approximately two-thirds of these cases, which included 62 patients with abnormal objective checklist results (UC: 17; CD: 45) (Table 2) and 21 additional patients (UC: 7; CD: 14) who were referred with complaints as a precautionary measure, but without objective findings. Although further diagnostic evaluation in the latter group was not mandated by the RAMP, these patients were included here for the sake of comprehensive patient monitoring in light of the serious nature of PML.

From the 83 patients, the IAC recommended brain MRIs in 56 patients (Table 3). The MRI results were inconsistent with PML, with a limited number of patients scoring 1-2 on the radiographic scale in the charter, and no individual patient having a score of 3 or higher. Diagnostic evaluation in 5 patients included lumbar puncture with PCR analysis of CSF for JC virus (UC: 2; CD: 3), all of which were
Table 3 Summary of RAMP algorithm results

\begin{tabular}{llll}
\hline & UC & CD & Total \\
\hline${\text { Patients }[n(\%)]^{\mathrm{a}}}$ & $n=1114$ & $n=1770$ & $n=2884$ \\
Referred to a neurologist & $24(2)$ & $58(3)$ & $82(3)$ \\
MRI performed & $15(1)$ & $41(2)$ & $56(2)$ \\
IAC involved & $24(2)$ & $59(3)$ & $83(3)$ \\
Lumbar puncture & $2(<1)$ & $3(<1)$ & $5(<1)$ \\
CSF analysed by PCR for JC viral DNA & $2(<1)$ & $3(<1)$ & $5(<1)$ \\
JC viral DNA detected by PCR in CSF & 0 & 0 & 0 \\
Diagnosed with PML by the IAC & 0 & 0 & 0 \\
\hline
\end{tabular}

CSF cerebrospinal fluid, IAC Independent Adjudication Committee, MRI magnetic resonance imaging, $P C R$ polymerase chain reaction, $P M L$ progressive multifocal leukoencephalopathy, $U C$ ulcerative colitis, $C D$ Crohn's disease, RAMP Risk Assessment and Minimisation for PML

${ }^{\text {a } P r o p o r t i o n s ~ a r e ~ b a s e d ~ o n ~ t h e ~ n u m b e r ~ o f ~ p a t i e n t s ~ w h o ~ c o m p l e t e d ~ o n e ~ o r ~ m o r e ~ s u b j e c t i v e ~ c h e c k l i s t s ~ a n d ~ w h o ~}$ had complete information in the RAMP algorithm section of the case report form 
negative. Given the fact that lumbar puncture is an invasive procedure and a common penultimate step in confirming PML, case history narratives for those five patients are provided in Electronic Supplementary Material 1: Table S2. No PML cases were identified by the IAC through 14 March 2013. Vedolizumab was approved by the US FDA and the European Medicines Agency in May 2014, and received over 50 approvals in countries around the world thereafter.

\subsection{Post-Study Follow-Up (Up to 2 Years Per Patient at 6-Month Intervals)}

As of March 2013, 1382 of 1794 (77\%) patients provided responses to at least one post-study telephone follow-up survey. For the 24-months post-study time point, data from 562 patients (67\% of eligible patients) were available. There were no reports of symptoms or signs referable to a PML diagnosis during this period.

\section{Discussion}

Because no validated method exists to screen for PML, the RAMP was devised as a clinical trial tool to address this potential risk. It consisted of a comprehensive evaluation, which included (1) exclusion of patients at higher risk of PML; (2) exclusion of patients in whom PML diagnosis might be more difficult to ascertain; (3) monitoring all patients for their risk of PML throughout their duration of the study using screening questionnaires; (4) objective testing; (5) a sequential case evaluation algorithm; and (6) post-study telephone follow-up.

Although sequential MRI scans are helpful in the diagnosis of PML when patients are still asymptomatic, early symptoms are the most practical means for early detection of the onset of PML. Because early diagnosis has been demonstrated to improve the likelihood of survival with less permanent neurological injury, we considered the use of early and frequent screening via questionnaires an important objective [24].

Neurological symptoms are common and occur more frequently in the IBD population than in the general population [25], as do white matter lesions on MR imaging [26]. This fact could theoretically confound the diagnosis of PML in this population. Moreover, IBD patients are usually cared for by gastroenterologists rather than neurologists, a fact that could make timely diagnosis of drugassociated PML more challenging. As a result, it was not evident from the outset how to create a system that is both sensitive enough to detect PML cases early in their course, while maintaining specificity in the face of many transient neurological symptoms that are inconsistent with PML. It was also not known if non-neurologists would respond well to the trainings and gain comfort with the subjective and objective facets of testing for a neurological disease. The types of symptoms and signs indicative of PML that were most likely to be detected by the questionnaires are not routinely evaluated by the cohort of investigators involved in the vedolizumab trials. Finally, the RAMP as a previously unvalidated algorithm, necessitated a high degree of compliance with risk minimisation procedures and associated timelines to assess its potential usefulness. It was not known at the outset what level of compliance might be achievable.

The overall experience with implementation of the RAMP across the clinical trial programme for vedolizumab revealed a low percentage of positive subjective checklists. Because PML is typified by new progressive neurological dysfunction, it seemed unlikely for symptoms consistent with PML to be missed based on the questions posed. Furthermore, gastroenterologists were able to successfully administer objective testing as qualitatively assessed by trainings, queries, source data verification, interaction with investigators, and compliance metrics, resulting in minimal demand for consultation with study neurologists. MRIs were performed in few patients overall, and even fewer outside of algorithm requirements.

A very small number of patients (5, approximately $0.2 \%$ of the study population) underwent lumbar puncture, the most invasive procedure necessitated by the RAMP for establishing PML diagnosis. As for MRIs, a lumbar puncture for CSF JCV analysis was not indicated by algorithm in all instances; three of these were performed electively by the local caring physician, and, in two of those instances, CSF had been collected for other standardof-care assessments. JC viral DNA was not detected in any CSF sample, and these negative findings (and the IAC's judgements) were confirmed by the fact that PML did not develop in any patients during the follow-up period.

One could speculate that baseline or longitudinal MRIs might have avoided the need for lumbar puncture in at least a few subjects; however, experience from the RAMP shows that the on-study MRIs were generally interpretable, even in the absence of a baseline examination. Moreover, performing thousands of potentially unhelpful MRIs to possibly prevent a very limited number of lumbar punctures argues against their clinical utility. For vedolizumab, with the evidence for gut selective biology and the absence of any proven PML events, MRIs would have represented an extremely low yield approach toward risk minimisation. For a drug with a very different pharmacologic or benefitto-risk profile, the relative merits of MRI monitoring could be considered as part of a tailored approach.

The data derived from this risk minimisation plan allow us to make useful inferences about the risk of PML in the 
vedolizumab-exposed population (the actual PML risk estimates from this dataset have been published elsewhere) [27]. The tools created and utilised are not vedolizumab or integrin antagonist specific. This fact lends strength and generalisability to other clinical development programmes where PML represents an actual or theoretical consideration. The checklists are simple and intuitive and do not require PML experts or neurologists to administer. The stepwise sequential evaluation mirrors clinical care and reserves more invasive testing for instances of higher clinical suspicion. Multiple checks and balances help ensure that new neurological symptoms are carefully followed and their evolution assessed in a timely manner. Given the rapidly progressive nature of clinical PML, it was important that the RAMP worked efficiently because delays in PML diagnosis could result in serious disability or death. In this global clinical trial programme with patients on five continents, adjudication was achieved within 7 days for approximately two-thirds of the cases; others required more time, often due to operational constraints (e.g. translation of medical documents, technical challenges with obtaining MRI scans, etc.). Although this was simply an assumption when the RAMP was devised, recent data suggest that early diagnosis of PML results in better outcomes [28]. Thus, the potential for investigators to receive expert feedback on PML likelihood and appropriate intervention measures within days is highly desirable. Patient and study staff education, training, and realtime support played an important role in clarifying the likelihood of development of this potentially devastating yet difficult-to-diagnose disease process, allowing the clinical development programme for vedolizumab to progress.

A limitation of the RAMP is the difficulty in ascertaining the predictive value of measures put in place in the absence of PML events. One option would be to utilise the RAMP or appropriate adaptation in a context with a better understood background rate of PML. Another limitation is the fact that the RAMP was performed uniquely in the clinical trial setting. The overwhelming amount of immunosuppressive use occurs in a real-world setting without case report forms, checklists or other systematic data collection methods. Some elements of the RAMP, e.g. elements of the informed consent language or educational materials, could potentially be adapted to serve real-world needs; however, pragmatic considerations could limit the utility of certain other RAMP components. While it is possible that PML cases could have gone undetected in spite of methods described herein, the authors have concluded that the extent of awareness and data collection via the RAMP, coupled with the progressive nature of PML, makes this possibility unlikely. Of note, since the approval of vedolizumab in many parts of the world in 2014, no cases of PML have been spontaneously reported, or otherwise identified, as of the submission of this manuscript [29].

\section{Conclusions}

The RAMP programme reported here demonstrates practical and effective screening and surveillance measures that can be used in clinical trials to manage potential PML risks of investigational or approved treatments. Implementation of the RAMP in a large global clinical trial programme was comprehensive and achievable, with high levels of compliance. No cases of PML were detected with vedolizumab in a patient population with at least some risk factors, suggesting a low likelihood of any relationship between vedolizumab mechanism of action and PML risk. Many elements of this plan could be utilised in other clinical trial programmes. Certain RAMP elements could be adapted for use in a real-world setting, however other measures are unlikely to be feasible or warranted. The RAMP cannot be validated in the absence of PML events; thus, future work should include assessing this programme in conjunction with drugs with proven PML risk. Additional work might include assessment of the RAMP by patients and healthcare professionals.

Acknowledgements Publication management support was provided by Peter Kreil, PhD, Takeda Pharmaceuticals International AG, Zurich, Switzerland.

Author Contributions All authors contributed to the design of the RAMP programme, provided critical review of the manuscript during its development, and approved the final version of the article.

\section{Compliance with Ethical Standards}

This manuscript complies with ethical standards. It has been approved by the Ethics Committee and has therefore been performed in accordance with the ethical standards laid down in the 1964 Declaration of Helsinki and its later amendments.

Conflict of interest Asit Parikh, Kristin Stephens, Irving Fox, Catherine Milch, Serap Sankoh, Jesse Shick, Mark Patti, and Megan McAuliffe are or were employees of Takeda Pharmaceuticals at the time this study was conducted. Megan McAuliffe owns stock/stock options of Biogen and was employed by Biogen at the time this manuscript was submitted. Michael H. Lev is a consultant for GE, MedyMatch, D-Pharm and Takeda. Jesse Shick owns shares in Takeda Pharmaceuticals. Joseph R. Berger reports grants and personal fees from Biogen, grants from TEVA, and personal fees from Genentech/Roche, Genzyme, Millennium/Takeda, Novartis, Inhibikase, ExcisionBio, Roche, Amgen, AstraZeneca, Alkermes and Bayer. David B. Clifford is supported by National Institutes of Health (NIH) grants NS077384, AI69495, NR012907, NR014449, NR012657 and UL1 TR000448, and by the Alzheimer Association, and has received research support from Eli Lilly, Roche and Janssen. He has provided scientific advisory or consulting to Amgen, Biogen, Dr. Reddy, Inhibikase, Genzyme/Sanofi, Takeda/Millennium, EMD 
Sorono, Roche/Genentech, Novartis, GSK, BMS, Pfizer, Quintiles, Drinker Biddle and Reath (PML Consortium Scientific Advisory Board), Shire, and Wave. As a member of the PML IAC, Eugene Major served as an unpaid advisor until he left US government service in 2014. James Provenzale has no disclosures relevant to the content of this manuscript.

Patient consent Prior to study enrolment, informed consent regarding a potential risk of PML associated with administration of the study drug was obtained. The text of the informed consent form can be found in Electronic Supplementary Material 2.

Funding Development of the RAMP and the clinical studies in which it was applied were sponsored by Millennium Pharmaceuticals, Inc., a wholly owned subsidiary of Takeda Pharmaceutical Company Limited.

Open Access This article is distributed under the terms of the Creative Commons Attribution-NonCommercial 4.0 International License (http://creativecommons.org/licenses/by-nc/4.0/), which permits any noncommercial use, distribution, and reproduction in any medium, provided you give appropriate credit to the original author(s) and the source, provide a link to the Creative Commons license, and indicate if changes were made.

\section{References}

1. Antonsson A, Green AC, Mallitt KA, O'Rourke PK, Pawlita M, Waterboer T, et al. Prevalence and stability of antibodies to the BK and JC polyomaviruses: a long-term longitudinal study of Australians. J Gen Virol. 2010;91(18):1849-53.

2. Knowles WA, Pipkin P, Andrews N, Vyse A, Minor P, Brown DW, et al. Population-based study of antibody to the human polyomaviruses $\mathrm{BKV}$ and $\mathrm{JCV}$ and the simian polyomavirus SV40. J Med Virol. 2003;71(1):115-23.

3. Schmedt N, Andersohn F, Garbe E. Signals of progressive multifocal leukoencephalopathy for immunosuppressants: a disproportionality analysis of spontaneous reports within the US Adverse Event Reporting System (AERS). Pharmacoepidemiol Drug Saf. 2012;21(11):1216-20.

4. Astrom KE, Mancall EL, Richardson EP Jr. Progressive multifocal leuko-encephalopathy; a hitherto unrecognized complication of chronic lymphatic leukaemia and Hodgkin's disease. Brain. 1958;81(1):93-111.

5. Campath (alemtuzumab) injection for intravenous use: US prescribing information. Cambridge: Genzyme Corporation; 2014.

6. Arzerra (ofatumumab) injection, for intravenous use: US prescribing information. East Hanover: Novartis Pharmaceuticals Corporation; 2016.

7. Adcetris (brentuximab vedotin) for injection, for intravenous use: US prescribing information. Bothell: Seattle Genetics, Inc.; 2016.

8. CellCept Intravenous (mycophenolate mofetil hydrochloride for injection): US prescribing information. South San Francisco: Genentech US, Inc.; 2015.

9. Rituxan (rituximab) injection, for intravenous use: US prescribing information. South San Francisco: Genentech, Inc.; 2016.

10. Tecfidera (dimethyl fumarate) delayed-release capsules, for oral use: US prescribing information. Cambridge: Biogen Inc.; 2016.

11. Berger JR. Progressive multifocal leukoencephalopathy and newer biological agents. Drug Saf. 2010;33(11):969-83.

12. Major EO. Progressive multifocal leukoencephalopathy in patients on immunomodulatory therapies. Annu Rev Med. 2010;61:35-47.
13. Piccinni C, Sacripanti C, Poluzzi E, Motola D, Magro L, Moretti $\mathrm{U}$, et al. Stronger association of drug-induced progressive multifocal leukoencephalopathy (PML) with biological immunomodulating agents. Eur J Clin Pharmacol. 2010;66(22):199-206.

14. Langer-Gould A, Atlas SW, Green AJ, Bollen AW, Pelletier D. Progressive multifocal leukoencephalopathy in a patient treated with natalizumab. N Engl J Med. 2005;353(4):375-81.

15. Van Assche G, Van Ranst M, Sciot R, Dubois B, Vermeire S, Noman M, et al. Progressive multifocal leukoencephalopathy after natalizumab therapy for Crohn's disease. N Engl J Med. 2005;353(4):362-8.

16. Kleinschmidt-DeMasters BK, Tyler KL. Progressive multifocal leukoencephalopathy complicating treatment with natalizumab and interferon beta-1a for multiple sclerosis. $\mathrm{N}$ Engl $\mathrm{J}$ Med. 2005;353(4):369-74.

17. Carson KR, Focosi D, Major EO, Petrini M, Richey EA, West $\mathrm{DP}$, et al. Monoclonal antibody-associated progressive multifocal leucoencephalopathy in patients treated with rituximab, natalizumab, and efalizumab: a Review from the Research on Adverse Drug Events and Reports (RADAR) Project. Lancet Oncol. 2009;10(8):816-24.

18. Li YY, Perez HD, Zollner TM. Fatalities in natalizumab treatment-a 'no go' for leukocyte recirculation approaches? Expert Opin Ther Targets. 2005;10(4):489-99.

19. Soler D, Chapman T, Yang LL, Wyant T, Egan R, Fedyk ER. The binding specificity and selective antagonism of vedolizumab, an anti-alpha4beta7 integrin therapeutic antibody in development for inflammatory bowel diseases. J Pharmacol Exp Ther. 2009;330(3):864-75.

20. Fedyk ER, Wyant T, Yang LL, Csizmadia V, Burke K, Yang H, et al. Exclusive antagonism of the $\alpha 4 \beta 7$ integrin by vedolizumab confirms the gut-selectivity of this pathway in primates. Inflamm Bowel Dis. 2012;18(11):2107-19.

21. Berger JR, Aksamit AJ, Clifford DB, Davis L, Koralnik IJ, Sejvar JJ, et al. PML diagnostic criteria: consensus statement from the AAN Neuroinfectious Disease Section. Neurology. 2013;80(25):1430-8.

22. Rudick RA, O'Connor PW, Polman CH, Goodman AD, Ray SS, Griffith NM, et al. Assessment of JC virus DNA in blood and urine from natalizumab-treated patients. Ann Neurol. 2010;68(3):304-10.

23. Plavina T, Subramanyam M, Bloomgren G, Richman S, Pace A, Lee $\mathrm{S}$, et al. Anti-JC virus antibody levels in serum or plasma further define risk of natalizumab-associated progressive multifocal leukoencephalopathy. Ann Neurol. 2014;76(6):802-12.

24. Dong-Si T, Gheuens S, Gangadharan A, Wenten M, Philip J, McIninch J, et al. Predictors of survival and functional outcomes in natalizumab-associated progressive multifocal leukoencephalopathy. J Neurovirol. 2015;21(6):637-44.

25. Morís G. Inflammatory bowel disease: an increased risk factor for neurologic complications. World J Gastroenterol. 2014;20(5):1228-37.

26. Geissler A, Andus T, Roth M, Kullmann F, Caesar I, Held P, et al. Focal white-matter lesions in brain of patients with inflammatory bowel disease. Lancet. 1995;345(8954):897-8.

27. Colombel JF, Sands BE, Rutgeerts P, Sandborn W, Danese S, D'Haens G, et al. The safety of vedolizumab for ulcerative colitis and Crohn's disease. Gut. 2017;66(5):839-51.

28. Dong-Si T, Richman S, Wattjes MP, Wenten M, Gheuens S, Philip J, et al. Outcome and survival of asymptomatic PML in natalizumab-treated MS patients. Ann Clin Transl Neurol. 2014;1(10):755-64.

29. Parikh A, Leach T, Wyant T, et al. Vedolizumab for the treatment of active ulcerative colitis: a randomized controlled phase 2 doseranging study. Inflamm Bowel Dis. 2012;18(8):1470-9. 
30. Parikh A, Fox I, Leach T, et al. Long-term clinical experience with vedolizumab in patients with inflammatory bowel disease. Inflamm Bowel Dis. 2013;19(8):1691-9.

31. Feagan BG, Rutgeerts P, Sands BE, et al. Vedolizumab as induction and maintenance therapy for ulcerative colitis. N Engl J Med. 2013;369(8):699-710.

32. Sandborn WJ, Feagan BG, Rutgeerts P, et al. Vedolizumab as induction and maintenance therapy for Crohn's disease. N Engl J Med. 2013;369(8):711-21.

33. Sands BE, Feagan BG, Rutgeerts P, Colombel JF, Sandborn WJ, Sy R, D'Haens G, Ben-Horin S, Xu J, Rosario M, Fox IH, Parikh
A, Milch C, Hanauer SB. Effects of vedolizumab induction therapy for patients with Crohn's disease in whom tumor necrosis factor antagonist treatment. Gastroenterology 2014;147(3):618-27.

34. Loftus EV Jr, Colombel JF, Feagan BG, et al. Long-term efficacy of vedolizumab for ulcerative colitis. J Crohns Colitis. 2017;11(4):400-11.

35. Vermeire S, Loftus EV Jr, Colombel JF, et al. Long-term efficacy of vedolizumab for Crohn's disease. J Crohns Colitis. 2017;11(4):412-4. 